\title{
KOGNITION UND ÜBERSETZEN. ÜBER DAS ÜBERSETZEN VON KONZEPTEN
}

\author{
JOANNA KUBASZCZYK \\ Adam Mickiewicz University - Poznań
}

ABSTRACT. In the article a new attempt has been made to define what is the unit of translation and how to distinguish it. In her considerations the author bases on the achievements of cognitive linguistics, claiming that the translator doesn't translate words, phrases or texts but the content units which realises in the concepts of language. Thus, it becomes possible to distinguish the concepts as translation units a priori before actual taking up the translation. The training in distinguishing the concepts of language and analysing them becomes therefore an important element of translation.

\section{Einführung}

An der Frage, was eine Übersetzung ist und was sie zu leisten hat, was übersetzen heißt, und was eigentlich übersetzt wird, scheiden sich die Geister.

Im Anfang war das Wort. Das Wort stand auch im Mittelpunkt übersetzungswissenschaftlichen Überlegens. Die Meinung, daß vor allem Worte und erst dann Sätze oder andere Einheiten übersetzt werden, wird auch heute noch von manchen Übersetzungswissenschaftlern vertreten. So geht z.B. Elisabeth Markstein (1999:246f.) davon aus, daß das Wort bei der literarischen Übersetzung im Gegensatz zur pragmatischen Gebrauchsprosa die übersetzerische Grundeinheit ist, denn ein dichter literarischer Text sei ein Text ,in dem kein Wort entfernt werden darf, ohne daß die ästhetische Aussage verändert wäre“. Das Wort stellt in der Konzeption "ein komprimiertes Informationsangebot" (Neubert 1991:12, angeführt nach Markstein) dar. Daher müsse der/die Übersetzende vor der Aufnahme der eigentlichen Übersetzung ,zur ,inneren Form des Wortes vordringen". Daraus ergibt sich "wie von selbst", so Markstein, "vom Wort auszugehen und dann erst zum Satz zu kommen"

Auch bei den Übersetzungswissenschaftlern, die sich schon längst von dem Gedanken verabschiedet haben, daß Wörter übersetzt werden, schleicht sich doch diese Idee manchmal durch die Hintertür in Form der Forderung ein, man solle so „wortgetreu wie möglich“ übersetzen. 
Eine andere Auffassung, die sich in Opposition zu dieser Position entw1ckelt hat, ist die der Textübersetzung. Nicht Wörter also, sondern Texte sind den Vertretem dieser Auffassung zufolge der eigentliche Übersetzungsgegenstand. Grundlage dessen ist die Einsicht, daß „Übersetzen nicht auf der Ebene der langue, des Sprachsystems, sondern der parole, der Äußerungen in Textzusammenhängen geschieht" (Koller 1992:91). So schreibt z.B. J.C. Catford (1965:1): "Translation is an operation performed on languages: a process of substituting a text in one language for a text in another."

Eine ähnliche Meinung vertritt Fritz Paepcke (1986:32):

Übersetzen ist eine besondere Form von Sprachverwendung, bei der der Ubersetzer von grammatisch und lexikalisch gestalteten Texten ausgeht. Denn nicht Sprachen, sondern sprachlich formulierte Texte werden von der A-Sprache in Texte der Z-Sprache übersetzt.

Auch in neueren Arbeiten geht man oft vom Textbegriff aus, so definiert z.B. Schreiber (1993:36) den Begriff Übersetzung folgendermaßen: „Eine Übersetzung ist eine interlinguale Texttransformation, die auf hierarchisierten Invarianzforderungen beruht".

Aufgrund der Erkenntnis, daß Lexeme oder Wörter nicht isoliert übersetzt werden, sondem in textuellen Zusammenhängen, entstand der Begriff der Übersetzungseinheit. Die Übersetzungseinheit wird von A. Malblanc als «le plus petit segment de l'énoncé dont la cohésion des signes est telle qu'ils ne doivent pas être traduis séparément» (1967:16) definiert (zit. nach Dzierżanowska 1990:105). O. Kade definiert die Übersetzungseinheit wie folgt:

Die Ubersetzungseinheit ist das jeweils kleinste Segment des AS-Textes, für das dank der potentiellen Äquivalenzbedingungen ein Segment im ZS-Text gesetzt werden kann, das die Bedingungen der Invarianz auf der Inhaltsebene erfullt. (1968:90, Hervorhebung J.K.)

Koller (1992:100) ist der Meinung, daß sprachstrukturelle Gegebenheiten bei der Bestimmung der Übersetzungseinheiten eine Rolle spielen. Je ähnlicher die Sprachen strukturell sind, desto kleiner seien die Übersetzungseinheiten.

M.E. ist es problematisch, daß erst nachdem eine die potentiellen Äquivalenzbedingungen erfüllende ZS-Substitution für das AS-Segment gefunden worden ist, bestimmt werden kann, was die Übersetzungseinheit in der AS ist. Man betrachte folgende Beispiele:

dt. jdn. Mores lehren - pln. nauczyć kogoś moresu - frz. apprendre à vivre à quelqu'un

Bei der Übersetzung aus dem Deutschen ins Polnische hätten wir drei Übersetzungseinheiten - jdn. - kogoś / Mores - mores / lehren - nauczyć, bei der Übersetzung ins Französische dafür nur eine Übersetzungseinheit, da es sich hier um eine Redewendung handelt. Ähnliches verdeutlichen weitere 
Beispiele, wo man je nach Z-Sprache nach dieser Konzeption entweder Wort für Wort oder ganzheitlich übersetzen müßte:
pln. stara panna - frz. vieille fille (stara panna) - dt. alte Jungfer - engl. spinster
engl. square root - dt. Quadratwurzel -- pln. pierwiastek kwadratowy
dt. positive Zahl - engl. positiv number - pln. liczba dodatnia

Eine solche Herangehensweise ist m.E. insofern falsch, als erst ausgegliedert werden muß, was zusammengehört, damit eine angemessene Entsprechung (ein Äquivalent) in der Z-Sprache gefunden werden kann. Wenn das nicht geschieht, entgeht der unerfahrene Übersetzer kaum der Gefahr, Wort fuir Wort zu übersetzen, vor allem wenn er in die Fremdsprache übersetzt und sich nicht auf das intuitive Sprachvermögen, auf sein Sprachgefühl, stützen kann. Diese Gefahr wird oft verkannt, weil sie sich gerade bei den relativ kleinen Einheiten aktualisiert und manifest wird, so etwa bei den Wortkollokationen. Redewendungen werden in der Regel bereits in der A-Sprache als eine Übersetzungseinheit erkannt und ausgegliedert (allerdings gilt das oft nicht für nicht fortgeschrittene Sprachlerner, die offensichtlich sehr häufig auch Redewendungen Wort für Wort übersetzen).

Einer solchen Definition der Übersetzungseinheit wie die o.a. liegt m.E. latent die Auffassung zugrunde, daß Wörter doch übersetzt werden können (zum Beispiel im terminologischen Bereich), und erst wenn das nicht möglich ist, weil in der Z-Sprache kein zufriedenstellendes Ergebnis erzielt werden kann, soll man sich auf die nächste Ebene hinbewegen (das Syntagma, den Satz, eine größere Texteinheit). Dies verdeutlicht zudem die Überzeugung von einigen Verfechtern der Textübersetzungstheorie, daß manchmal auch ein Wort Text sein könne.

An der Definition der Übersetzungseinheit von $\mathbf{O}$. Kade wäre auch zu bemängeln, daß sie einsprachig ausgerichtet ist, weil das zielsprachliche "Ergebnis" der Umsetzung in der Beschreibung nicht berücksichtigt wird. Diese Vorgehensweise läßt unreflektiert, daß in der ZS eine Einheit beispielsweise durch ein Syntagma und nicht durch einen Satz oder ein Morphem ersetzt wird.

An der Stelle möchte ich die im weiteren Teil des Aufsatzes aufgestellte These vorwegnehmen, die lautet, daß Menschen in komplexen Bildern denken und daher auch nicht Wörter, sondern komplexe Bilder übersetzt werden. Diesem Umstand hat die Übersetzungswissenschaft m.E. auch Rechnung zu tragen. Wie die im weiteren Teil des Aufsatzes durchgeführte Analyse eines Übersetzungsvorgangs und seines Ergebnissses zeigen wird, läßt sich mit einer solchen Bestimmung der Übersetzungseinheit wie die oben angeführte auch deswegen wenig anfangen, weil sie die Struktur des menschlichen Denkens nicht mitreflektiert. 
Zunächst aber soll noch erwähnt werden, daß Koller unter denotativem Aspekt Übersetzungseinheiten auf der Wortebene, auf der Syntagma- und auf der Satzebene unterscheidet (1992:100). Der erste Fall ist die Übersetzungseinheit in Form eines Wortes, was im Bereich der Terminologie gelten soll. Koller illustriert das mit folgenden Beispielen:

\section{dt. Umsatzvolumen $\rightarrow$ frz. volume de ventes \\ $d t$. Stromkreis $\rightarrow$ engl. electric circuit}

Ein Syntagma kann eine Übersetzungseinheit im Bereich der Terminologie (engl. dataprocessing - dt. Datenverarbeitung) und phraseologisch gebundener Ausdrücke (dt. blinder Passagier - engl. stowaway) sein, im Bereich redensartlicher Ausdrücke (ins Gras beißen), Floskeln (am Rande bemerkt) und stereotyper Formulierungsmuster (in Erkenntnis der Bedeutung). Ein Satz ist nach Koller eine Übersetzungseinheit im Falle von Sprichwörtern und normativ festgelegten Ausdrücken und Formeln. Ein Text kann eine Übersetzungseinheit sein, wenn es sich um einen poetischen Text oder einen Werbetext handelt.

Wenn die Definition der Übersetzungseinheit von $\mathrm{O}$. Kade für die Praxis des Übersetzens wenig tauglich ist, muß man weiter fragen, wie eine Übersetzungseinheit betimmt werden soll. Anscheinend werden weder Wörter noch Texte übersetzt, zumal der Begriff des Textes so allgemein ist, daß man fragen müßte, wo ein Text aufhört und der andere beginnt.

\section{Übersetzung von Konzepten}

Die Grundannahme dieser Erörterung, die ich im weiteren Teil dieses Aufsatzes zu begründen versuche, ist, daß weder Wörter noch Texte und auch nicht Sprachen, sondern in Konzepten festgehaltene kognitive Inhalte übersetzt werden. Kognitive Inhalte widerspiegeln das Weltbild eines Menschen, seine Weltsicht und die Art und Weise, wie er kognitive Erkenntnisse ordnet. Die hier präsentierte Auffassung geht auf theoretische Erkenntnisse der kognitiven Linguistik zurück und versucht sie für die Übersetzungswissenschaft fruchtbar zu machen. Interessanterweise finden wir diese Ansicht bereits in älteren übersetzungswissenschaftlichen Ansätzen, die aber wenig beachtet wurden. So definiert W. Winter (1961:68) das Übersetzen wie folgt: „To translate is to replace the formulation of one interpretation of a segment of the universe around us and within us by another formulation as eqivalent as possible".

Kognitive Inhalte werden nach Langacker (vgl. Tabakowska 1995) in der jeweiligen Sprache als Inhaltseinheiten (content units) konzeptualisiert. Die Einheiten haben einen symbolischen Charakter. Das Ergebnis der Konzeptualisierung sei hier als Konzept bezeichnet. Dabei soll zwischen Gesamtkonzepten 
(globalen, umfassenden, komplexen Konzepten) und Teilkonzepten (detaillierten Konzepten) unterschieden werden. Das Gesamtkonzept umfaßt und ordnet die ganze Aussage. Im traditionellen Sinne könnten wir sagen, daß das Gesamtkonzept sich auf den ganzen Text bezieht. Die Teilkonzepte sind dem Gesamtkonzept untergeordnet, was mehrstufig verlaufen kann, z.B.:

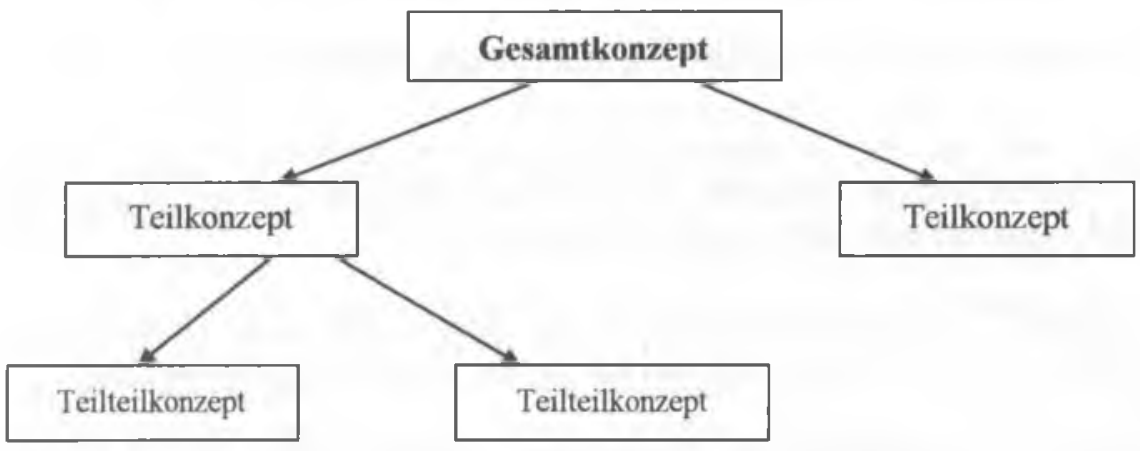

Eine solche Betrachtungsweise einer Inhaltseinheit stimmt m.E. mit der Auffassung von Langacker (1987:198) überein, der betont, daß eine Einheit (entity) sehr allgemein $\mathrm{zu}$ verstehen ist, denn der Begriff bezieht sich auf jede konzeptuelle Struktur (,,anything we might conceive of or refer to..."), unbeachtet ihrer inneren Komplexität. So kann ein komplexes Konzept Bestandteil einer Relation auf einer höheren Organisationsstufe werden, wodurch sein eigenes internes Profil durch das Profil einer Relation höheren Grades bestimmt wird (vgl. Turewicz 1998:68).

Kognitive Inhalte können in der Ausgangssprache und in der Zielsprache anders konzeptualisiert werden. Die Konzeptualisierung erfolgt dadurch, daB auf kognitive Inhalte Schemata (schemas) auferlegt werden. Durch die Auferlegung von Schemata werden kognitive Inhalte organisiert und voneinander abgegrenzt. Man könnte also als Arbeitshilfe folgende Definition aufstellen:

Ubersetzen heißt ein in der AS ausgedrücktes Konzept durch ein zielsprachliches Konzept zu ersetzen, indem auf die dem AS-Konzept zugrundeliegenden kognitiven Inhalte zielsprachliche Organisationsschemata auferlegt werden, wobei die Identität oder maximale Ähnlichkeit der Konzepte anzustreben ist.

Die hierarchische Anordnung der Konzepte hat dabei zur Folge, daß Teilkonzepte in der Zielsprache ,austauschbar" sind, wenn sie in das Gesamtkonzept nicht passen. Das erfordert eine globale Betrachtungsweise oder, wie Wilss (1988:VIII) sie nennt, eine übersetzerische Gesamtplanung, ,in der alle übersetzungsprozessual relevanten Dimensionen kognitiv vermessen werden, um den 
für das Erreichen des übersetzerischen Gesamtzieles möglichst günstigen Navigationspfad zu ermitteln".

Hierarchische Anordnung von Konzepten bedeutet auch, daß komplexe Konzepte und nicht detaillierte Konzepte vorrangig übersetzt werden.

Dies soll an einigen Beispielen verdeutlicht werden. Nehmen wir an, jemand hat folgende Aussage ins Deutsche und ins Englische zu übersetzen:

pln. Masz chusteczkę do nosa? Tak, mam chusteczkę higienicznq.

Wollte er sich der oben kritisierten Auffassung von Kade/Koller in bezug auf die Übersetzungseinheit bedienen, so wüßte er wohl nicht, ob er die Aussage wie im Beispiel (a) oder im Beispiel (b) einteilen sollte:

(a) masz $\mid$ chusteczkę $\mid$ do $\mid$ nosa $\mid$ tak $\mid$ mam |chusteczkę |higieniczna|

(b) masz |chusteczkę do nosa | tak | mam |chusteczkę higieniczna|

Erst nach der Fertigstellung der Übersetzung, nachdem er das richtige Äquivalent gefunden hat, weiß er, daß die zweite Einteilung sinnvoller ist. Geht er aber nach Konzepten vor, dann weiß er gleich daß chusteczka do nosa ein Konzept ist und chusteczka higieniczna ein anderes und daß die den Konzepten zugrundeliegenden kognitiven Inhalte als ein Ganzes behandelt werden müssen. So sucht er für den kognitiven Inhalt [ein (meist) aus Stoff oder Papier gemachter Gegenstand in (meist) quadratischer Form zum Naseputzen], der im Polnischen als chusteczka do nosa konzeptualisiert wird, ein entsprechendes Konzept im Deutschen und im Englischen:

$\rightarrow d t$. (Wahrig: kleines Lein-, Batist- oder Seidentuch zum Naseschneuzen)

$\rightarrow$ Taschentuch

$\rightarrow$ engl. Longman: a piece of cloth or thin soft paper for drying the nose, eyes, etc.)

$\rightarrow$ (pocket) handkerchief

Bei dem Konzept chusteczka higieniczna geht unser potentieller Übersetzer analog vor: (ein aus Papier gemachter Gegenstand in quadratischer Form zum Naseputzen)

$\rightarrow$ dt. Papiertaschentuch / Tempo(taschentuch)

$\rightarrow$ engl. a paper handkerchief

Die Einteilung wie im vorstehenden (b)-Beispiel entspricht der Einteilung nach Konzepten. Wie oben angedeutet, werden kognitive Inhalte mit Hilfe von Schemata geordnet. Da die Schemata in verschiedenen Sprachen anders sein 
können, werden die Konzepte auch anders geordnet. Eine Erörterung dieser Frage würde jedoch den Rahmen dieses Aufsatzes sprengen.

(pln.) $\rightarrow$ masz $\mid$ chusteczke do nosa $\mid$ tak $\mid$ mam $\mid$ chusteczke higieniczna $\mid$ $\rightarrow(d t.) \rightarrow$ hast du $\mid$ ein Taschentuch $|j a|$ ich habe $\mid$ ein Tempo(taschentuch) $\mid$ (engl.) $\rightarrow$ do you have $\mid$ a handkerchief $\mid$ yes I do|I have a paper one

Die Übersetzung unseres Beispielsatzes würde demzufolge so aussehen:

$\rightarrow$ Hast du ein Taschentuch? Ja. ich habe ein Tempo(taschentuch).

Einen ähnlichen Grundgedanken, ohne allerdings über Konzepte zu sprechen, entwickelte Nida und Vertreter seiner Schule (z.B. Larson 1984) - ich referiere hier in Anlehnung an Kußmaul (1999:49). Ihre Übersetzungsmethode besteht darin, daß nicht Wörter, sondern Bündelungen semantischer Merkmale übersetzt werden (Vgl. Nida 1974:46). Infolge des Zurückgreifens auf eine derartige Übersetzungsmethode ,lösen wir uns in einem Abstraktionsschritt notwendigerweise von der ausgangssprachlichen Wortform und können dann die abstrahierte Bedeutung in die Gefäße anderer zielsprachlicher Formen - häufig Paraphrasen - gießen“ (Kußmaul 1999:49).

Auffallend ist es, daß diese Vorgehensweise auch im Spracherwerb funktioniert. So würde ein Sprachlemer, gleichgültig, ob es sich um seine Muttersprache oder um eine Fremdsprache handelt, falls er die (angemessene) Bezeichnung (ein Konzept) für einen kognitiven Inhalt nicht kennt bzw. das Konzept ihm gerade entfallen ist, höchstwahrscheinlich beschreibend vorgehen und fragen:

Wie heißt das nochmal, so ein (viereckiger) Gegenstand zum Naseputzen. (meist) aus Batist oder Papier (gemacht)?

Das Aussondem von Konzepten und die Übersetzung von Konzepten und nicht von Wörtern oder Übersetzungseinheiten im Sinne von Kade/Koller zeigt ihre praktische Anwendbarkeit auch bei komplexeren sprachlichen Einheiten, die aber ein einziges Konzept in Form einer Konstruktion sind. Will man im Deutschen den kognitiven Inhalt [riesigen Hunger haben] zum Ausdruck bringen, kann man sich folgender Tier-Metapher bedienen:

\section{dt. Hunger wie ein Bär haben}

Im Polnischen wird der kognitive Inhalt gleichfalls mit einer Tier-Metapher ausgedrückt, allerdings assoziert man Hunger mit einem anderen Tier:

pln. być glodnym jak wilk ${ }^{1}$

${ }^{1}$ Was wiederum auch im Deutschen geht, vgl. Wolfshunger, Hunger wie ein Wolf haben. Im Slownik frazeologiczny jezyka polskiego kann man noch einen anderen Vergleich finden, dh. być glodnym jak pies, aber dieser Vergleich scheint weniger verbreitet zu sein. 
Der Sachverhalt läßt sich auch noch an einem anderen Beispiel verdeutlichen, dem ein sehr komplexer kognitiver Inhalt zugrunde liegt. In einem Zeitungsartikel Zum Kochen keine Zeit (FAZ, 20.08.1998) finden wir folgende Aussage:

Die Zeit für das aufwendige Kochen scheint immer geringer bemessen zu sein.

Der kognitive Inhalt des Gesamtkonzeptes ist [man hat immer weniger Zeit, daher kann man auch nicht kochen], der kognitive Inhalt des Teilkonzeptes gering bemessen ist [wenn eine Sache knapp bemessen ist, z.B. Stoff, dann ist es schwierig, etwas daraus zu machen; wenn ein Schneider ein immer kleineres Stück Stoff zur Verfügung bekommt, dann kann er kaum etwas daraus schneidern]. So ergibt die Analyse des dem AS-Konzept zugrundeliegenden kognitiven Inhalts folgendes Konzept im Polnischen:

Coraz trudniej wykroić czas na (czasochlonne, absorbujqce) gotowanie.

$\mathrm{Da}$ kognitive Inhalte übersetzt werden, die in sprachlichen Konzepten festgehalten werden, und daß man daher Konzepte als Übersetzungseinheiten betrachten muß, sieht man auch am nächsten Beispiel:

Ten, kto jest zmęczony, może iść spać.

In der aus sieben Wörtern bestehenden Aussage, haben wir eigentlich nur zwei kognitive Inhalte, also zwei Konzepte:

ten kto jest zmęczony | może iść spać

Somit können diese kognitiven Inhalte mit zwei verschiednen Konzepten im Deutschen wiedergegeben werden. Die Konzepte variieren, je nachdem, welche Schemata an die Inhalte geknüpft werden.

$\rightarrow$ Wer müde ist | kann schlafen gehen

$\rightarrow$ Die Müden|können schalfen gehen etc.

Wenn kognitive Inhalte und nicht Wörter übersetzt werden, entgeht man der Gefahr von Homonymen:

czysty zysk - der reine Gewinn

czyste powietrze - reine /saubere Luft

czysta robota - saubere Arbeit

czysta szyba - saubere Fensterscheibe

czysty przypadek - purer / reiner Zufall

czysta wódka - Wodka pur / reiner Wodka 
Konzepte können die Form von einfachen (nicht zerlegbaren) Einheiten und zusammengesetzten Einheiten - Konstruktionen - annehmen. So sind z.B. einfache, nicht zerlegbare Einheiten solche Konzepte wie dom, karta, dt. gar, Haus, aber auch z.B. Redewendungen wie etwas auf die leichte Schulter nehmen. Die Konstruktionen entstehen infolge der Integration von zwei oder mehr Strukturen - domek, chusteczka do nosa, chusteczka higeniczna, Taschentuch etc. (vgl. Tabakowska 1995:16 und 95). An den Konstruktionen läßt es sich noch ablesen, aus welchen Elementen sie entstanden sind, z.B.:

chusteczka do nosa $\leftarrow$ chusteczka do (wycierania / do czyszczenia) nosa

Taschentuch $\leftarrow$ ein Tuch, das in der Tasche getragen wird domek $\leftarrow$ maly $[-e k]$ dom

Infolge der Übersetzung können folgende Ersetzungen vollzogen werden:

ein einfaches Konzept (engl. knit) $\rightarrow$ eine Konstruktion (pln. robić na drutach. dt. stricken ${ }^{2}$ )

ein einfaches Konzept (pln. dom) $\rightarrow$ ein einfaches Konzept (dt. Haus) eine Konstruktion (pln. mieć nadzieję, dt. hoffen) $\rightarrow$ ein einfaches Konzept (engl. hope)

eine Konstruktion (dt. Taschentuch) $\rightarrow$ eine Konstruktion (pln. chusteczka higieniczna)

Interessanterweise lassen auch die deklarierten Befürworter der Übersetzung von Wörtern in der Praxis bei der Beurteilung der Übersetzungsqualität den konzeptuellen Übersetzungen den Vortritt, ohne sich wohl immer dessen bewußt zu werden. So zeigt eingangs zitierte Elisabeth Markstein (1999:247), daß der Sinn der aus Gogols Mainacht stammenden Aussage „Tichi i pokojny eti prudy" nicht ausgeschöpft wird, wenn man sie wörtlich übersetzt (,Still und ruhig sind diese Teiche"), und die beste Übersetzung ist die von Michael Pfeiffer "Still und friedlich ruhen die Teiche", weil das Konzept des Friedlichen und des Ruhens hier hinübergerettet wird und der Satz auch rhythmisch erschlossen ist. Dies beweist nur, daß auch in der künstlerischen Prosa in der Praxis Gesamtkonzepte übersetzt werden, und nicht bloß Wörter (obwohl ja ein Wort u.U. durchaus auch ein Konzept sein kann).

\section{Überprüfung der Konzeption anhand eines Praxisfalls}

Im ersten Teil dieses Aufsatzes ist die These aufgestellt worden, daß weder Wörter noch Texte, sondern Konzepte übersetzt werden. Im weiteren soll jetzt diese These anhand einer Übersetzung überprüft werden.

${ }^{2}$ Das deutsche Verb stricken ist im Langackerschen Sinne als eine Konstruktion einzuordnen, denn es besteht aus zwei Elementen, zwei Bestandteilen [strick + en]. 
Ich habe mich bei der Wahl des Textes für einen relativ kurzen Text entschlossen, der eine Handlungsanleitung darstellt. Es ist ein authentisches deutsches Kochrezept, das ins Polnische übersetzt wurde. Der Übersetzung war die Bestimmung von Konzepten vorausgegangen. Sie erfolgte auf der Basis der Ausgangssprache. Die Frage war, was bei einer Einheit zu einem komplexen kognitiven Bild gehört. Ich ging davon aus, daß immer komplexe Konzepte und nicht „Minimalkonzepte" übersetzt werden. Das entspricht der oben erläuterten Meinung, daß man von oben nach unten gehen muB, also vom Globalkonzept zum Detailkonzept und nicht umgekehrt. Als die erste konzeptuelle Ebene ist daher das ganze Kochrezept definiert worden. Auf der zweiten Ebene sind die Auflistung von Zutaten und die Kochanleitung als globale Konzepte analysiert worden. Die Aussonderung dieser Konzepte ist insofern wichtig, als sie den Textaufbau bestimmen. Im nächsten Schritt nach unten wurden dann die zu übersetzenden Einzelkonzepte (Teilkonzepte) ausgesondert. Die nachstehend aufgelisteten Syntagmen entsprechen den Konzepten. Infolge der Analyse der ausgesonderten Konzepte ist folgendes festgestellt worden:

Die Anordnung der Angaben in der Zutatenliste stimmt mit den übersetzerischen Konzepten überein, d.h. eine Angabe gleicht einem Konzept. Konzepte in der Kochanleitung decken sich weitgehend mit einfachen Sätzen.

Anhand einiger Beispiele möchte ich im weiteren Teil eine solche Einteilung des Textes in Konzepte begründen.

\section{Crêpes mit Weinschaum} für 4-6 Portionen
Crêpes, krepy, naleśniki, naleśniki francuskie z musem winnym (pianką winnq̨) na 4-6 porcji
Teig

$40 \mathrm{~g} \mathrm{Mehl}$

20 g Stärkemehl

1 Vanillestange

4 Eier

etwas Salz

etwas Zucker

$100 \mathrm{~g}$ Sahne

$20 \mathrm{~g}$ Butter

etwas Butter für die Pfanne

Weinschaum

80 g Zucker

5 Eigelb

Saft einer Zitrone

$200 \mathrm{ml} \mathrm{Weißwein}$

außerdem

200 g Erdbeeren

Puderzucker zum Garnieren
Ciasto

4 dkg maki

2 dkg maki ziemniaczanej, kartoflanki,

1 laska wanilii

4 jajka

trochę soli

troche cukru

$10 \mathrm{dkg}$ śmietany

2 dkg masła

trochę masla na patelnie

mus winny

$8 \mathrm{dkg}$ cukru

5 żóltek

sok $\mathrm{z}$ jednej cytryny

$200 \mathrm{ml}$ białego wina

oprócz tego, poza tym

$20 \mathrm{dkg}$ truskawek

cukier puder do dekoracji,

przystrojenia, udekorowania 
Mehl und Stärkemehl in eine Schułssel geben

in die Mitte eine Vertiefung machen die Vanillenstange der Länge nach aufschneiden

das Mark mit dem Messer ausstreichen zum Mehl geben

Eier, Salz und Zucker in die Vertiefung geben

die Sahne angießen

alles zusammen mit einem Schneebesen glattrühren

die Butter in einer Pfanne bräunen

lauwarm zum Teig geben

und gut verrühren mạkę i mąkę ziemniaczaną / mạkę zwykłą i ziemniaczaną

wsypać / dać / wlożyć do miski

w środku zrobić zagłębienie / dołek

laskę wanilii przeciąc / naciąć wzdluż

miazssz wyskrobać nożem

dodać do mąki

jajka, sól i cukier wlożyć do zaglębienia

wlać śmietanę

wszystko razem ubić trzepaczką tak, aby nie bylo grudek

maslo zarumienić na patelni

dodać letnie do ciasta

i dobrze zmieszać
Zum Braten der Crêpes jeweils sehr wenig Butter in einer schweren Eisen- oder Stahlpfanne erhitzen

Dann gerade so viel Teig hineingeben, daß der Boden dunn bedeckt ist.

Eventuell uberschussigen Teig wieder in die Schüssel zurückgießen.

Die Crêpes auf der einen Seite backen, dann wenden.

Das Braten geht sehr schnell

Also Vorsicht, daß nichts anbrennt!
Do smażenia rozgrzać/roztopić na ciężkiej żelaznej albo stalowej patelni za każdym razem odrobinę masła,

a następnie nalać tyle ciasta, by przykryć cienką warstwą dno.

Ewentualny nadmiar ciasta odlać $\mathrm{z}$ powrotem do miski.

Naleśniki smażyć na jednej stronie, a następnie odwrócić.

Smażenie idzie bardzo szybko

więc uwaga, żeby nie przypalić!

\section{Die Crêpes}

jeweils sofort nach dem Fertigbacken im auf $100^{\circ} \mathrm{C}$ vorgeheizten Backofen auf einen großen Teller legen, der mit einem zweiten abgedeckt wird. So bleiben sie warm und trocknen nicht aus.
Gotowy naleśnik zaraz po usmażeniu ułożyć na dużym talerzu i wstawić do nagrzanego piekarnika $\left(100^{\circ} \mathrm{C}\right)$

a talerz przykryć drugim

dzięki temu naleśniki nie ostygną

i nie wyschna.
Für den Weinschaum Zucker, Eingelbe und Zitronensaft in einer Schüssel gut verrahren,

den Weißwein dazugeben.

Unter Rühren über einem heißen Wasser-

bad mit dem Schneebesen aufschlagen

bis die Masse steif geworden ist
Na mus dobrze wymieszać w misce cukier, żóltka i sok z cytryny, dodać biale wino ciagle mieszając, ubijać nad gorącą parą trzepaczka, aż masa będzie sztywna.
Die Erdbeeren waschen, entstielen und halbieren.
Truskawki umyć obrać z szypułek i przepolowić 
Die Crêpes auf sechs vorgewärmten Tellern anordnen,

mit dem noch warmen Weinschaum umgießen,

mit den Erdbeeren garnieren

und nach Belieben mit Puderzucker bestreuen.
Ułożyć naleśniki na sześciu podgrzanych talerzach

oblać je dookoła jeszcze ciepłym musem, udekorować truskawkami

i posypać wedlug upodobania cukrem pudrem

Das erste komplexe Konzept, das dem kognitiven Bild entspricht, ist die Konstruktion "Crêpes mit Weinschaum“. Diese Bezeichnung profiliert auf der kognitiven Basis Teigwaren/Crêpes eine spezielle Art von Pfannkuchen (Eierkuchen). Die ZS kann u.U. für diesen Sachverhalt, oder kognitiv ausgedrückt, für diese markierte Fläche, eine einzige Bezeichnung haben. Da es im Polnischen keine ganzheitliche Konzeptualisierung des kognitiven Inhalts gibt, der dem Konzept "Crêpes mit Weinschaum" zugrunde liegt, kann der Übersetzer sie entweder schaffen (z.B. wajnkrepy), oder diese Konstruktion in Teilkonzepte zerlegen. Die zwei Teilkonzepte sind zueinander in eine Relation gesetzt worden durch die Präposition mit, was zusätzliche Begründung ist, das Ganze als ein Konzept in Form einer Konstruktion zu betrachten.

Ein anderes komplexes Konzept ist die Angabe "Saft einer Zitrone“. Wiederum haben wir hier mit einem Bild zu tun, das automatisch mit einem anderen Bild und einem anderen Konzept - sok z jednej cytryny - ersetzt wird. Diese Vorgehensweise erklärt, warum wir auf einmal im Polnischen ein Zusatzelement "z" im Text stehen haben. Dem kognitiven Inhalt [Saft, der aus einer Obstsorte gepreßt wird] entspricht im Polnischen die Konstruktion sok z malin, jabłek, gruszek etc.

Es könnte die Frage gestellt werden, warum z.B. die Angabe „etwas Salz“ als ein Konzept betrachtet wird. Hier wurde wiederum auf eine unbestimmte Fläche ein Profil aufgelegt, wodurch aus einer unbestimmten Menge Salz ein Bereich ausgeschnitten worden ist. Man kann sich durchaus vorstellen, daß es eine Sprache gibt, wo es für „etwas Salz“ einen separaten Begriff gibt. Dasgleiche betrifft alle anderen Mengenangaben in diesem Text.

An dem ersten Konzept der Kochanleitung läßt sich absehen, wie die einzelnen Teilkonzepte sich gegenseitig bedingen. Wollten wir Teilkonzepte aussondern, so erhielten wir:

\section{Mehl und Stärkemehl| in eine Schüssel geben}

und noch eine Ebene tiefer:

Mehl | und |Stärkemehl| in |eine Schüssel |geben

Die Übersetzung aber erfolgt auf der höheren Ebene, wo das Konzept Mehl [etwas Lockeres, nicht Festes] das Konzept geben beeinflußt, womit wir im Polnischen das Konzept wsypać erhalten. Ähnlicherweise beinflussen sich die Konzepte Mehl und Stärkemehl, was es möglich macht, sie im Polnischen mit 
maka zwykla $i$ ziemniaczana wiederzugeben, um die Wiederholung zu vermeiden. Daß die Konzeptualisierung auf der Satzebene erfolgt, sehen wir auch daran, daß der Satzaufbau als Schema auf die anderen Elemente auferlegt wird und sie entsprechend ordnet.

\section{Schlußfolgerungen}

In diesem Aufsatz habe ich mich mit der Auffassung auseinandergesetzt, wonach Wörter bzw. Texte übersetzt werden und als Alternative das Übersetzen von Konzepten vorgeschlagen. Dieser Vorschlag hat $\mathrm{m}$.E. auch wichtige übersetzungsdidaktische Implikationen. Lemen künftige Übersetzer, Konzepte richtig zu erkennen und auszusondem, ist der übersetzerische Erfolg halbwegs gesichert. Dies betrifft vor allem die Fälle, wo man - traditionell gesprochen „wortgetreu" nicht übersetzen kann. Gibt es aber Fälle, wo man es kann?

\section{LITERATUR}

Catford, J.C.: A Linguistic Theory of Translation, London: Oxford Univ. Pr., 1965.

Dzierżanowska, H.: Przeklad tekstów nieliterackich. Na przykladzie języka angielskiego, Warszawa 1988.

Gallagher, J.D.: Möglichkeiten und Grenzen der Übersetzungsäquivalenz. In: Börner, W.; Vogel, K. (Hrsg.): Kontrast und Äquivalenz, Tübingen 1998.

Kade, O.: Zufall und Gesetzmäßigkeit in der Übersetzung, Leipzig 1968.

Koller, W.: Einführung in die Übersetzungswissenschaft, 1992.

Kuß3maul, P.: Semantik. In: Snell-Hornby/Hönig/Kußmaul/Schmitt (Hrsg.): Handbuch Translation, Tübingen 1999.

Markstein, E.: Erzählprosa. In: Snell-Hornby/Hönig/Kußmaul/Schmitt (Hrsg.): Handbuch Translation, Tübingen 1999.

Paepcke, F. : Im Übersetzen leben. Übersetzen und Textvergleich, Tübingen 1986.

Schreiber, M.: Übersetzung und Bearbeitung. Zur Differenzierung und Abgrenzung des Übersetzungsbegriffs, Tübingen 1993.

Tabakowska. E.: Gramatyka i obrazowanie. Wprowadzenie do językoznawstwa kognitywnego. PAN, Kraków 1995.

Winter, W.: Impossibilities of Translation. In: Arrowsmith, W., Shattuck, R. (Hrsg.): The Craft and Context of Translation. A Symposium, Austin/Texas 1961. 